\title{
Cervical spondylosis in paraplegic patients and analysis of the wheelchair driving action: A preliminary communication
}

\author{
Atsushi Kojima ${ }^{1}$, Akio Nakajima ${ }^{2}$ and Kenji Koyama ${ }^{3}$ \\ ${ }^{1}$ Department of Orthopaedic Surgery, Higashinagoya National Hospital, 5-101, Umemorizaka, Meitou-ku, Nagoya \\ 465; ${ }^{2}$ Department of Rehabilitation Medicine, Chubu Rosai Hospital, 1-10-6, Koumei, Minato-ku, Nagoya 455; \\ ${ }^{3}$ Rehabilitation Engineering Center for Employment Injuries, 1-10-5, Koumei, Minato-ku, Nagoya 455, Japan
}

\begin{abstract}
We investigated cervical spondylotic changes in paraplegic patients by examining the radiographs of their cervical spine. The incidence of cervical spondylosis in these patients was significantly higher than was found in normal control subjects of a matching age. We attributed this finding to the mechanical stress on the cervical spine during wheelchair driving.

We then performed a biomechanical analysis using electromyography and goniometer. Paraplegic patients frequently flexed and extended their cervical spine shown by the goniometer, whilst the normal control subjects walked almost without moving their cervical spine. However, the integrated electron myography (IEMG) of the paraplegic patients was not synchronously increased with the integration of goniometer (IGOM) and the IEMG of the normal controls was significantly larger than that found in paraplegic patients on increasing their speed. This was thought to be necessary for steadying the head whilst walking or running.
\end{abstract}

Keywords: paraplegia; cervical spondylosis; electromyography; wheel-chair use

\section{Introduction}

Paraplegic patients frequently develop clinical manifestations of cervical spondylosis (CS) a long time after injury. We thought that using a wheelchair is one of the etiologic factors related to this phenomenon. To confirm the high incidence of CS in paraplegic patients, we performed an epidemiological investigation in paraplegic patients and in normal control subjects, and also conducted an electromyographic analysis of the cervical muscles and a kinetic analysis of the cervical spine whilst they were propelling the wheelchair to clarify the effect of wheelchair driving on the cervical spine.

\section{Materials and methods}

\section{Epidemiological investigation}

We investigated the incidence of CS in 87 patients $(83$ men, four women) who had spinal cord damage extending from $\mathrm{T} 1$ to $\mathrm{L} 1$, and 200 healthy control subjects (128 men, 72 women). The age of paraplegic patients ranged from $23-78$ years, with a mean of 51.8 years. We sent the patients a questionnaire concerning symptoms of CS (eg, neck pain, numbness of upper extremities, a motor weakness), sports practice and time of wheelchair use. We then performed roentgen-

Correspondence: A Kojima ological studies of the cervical spine and of the injured spine. The relationships of the CS symptoms, the incidence of CS changes, age, level of injury, and the duration of paraplegia were then determined.

The age of the control group ranged from 15-74 years, with a mean of 39.7 years. The relationships between age and the incidence of CS changes on radiographs were determined. This group consisted of persons who came to our facility with a whiplash injury from a traffic accident; they subsequently had radiographs of their cervical spine. None had clinical findings of $\mathrm{CS}$.

\section{Biomechanical examination}

We conducted an electromyographic (EMG) analysis of the cervical muscles and a kinetic analysis with a goniometer of five asymptomatic paraplegic patients during wheelchair driving. These paraplegic patients had no CS changes on radiography. Our system is shown in Figure 1. EMG was performed for the sternocleidomastoid and trapezius muscles at a speed change of $4 \sim 8 \mathrm{~km} / \mathrm{h}$ and an inclination of $2 \sim 6 \%$ on a treadmill in accordance with a time sheet. We then obtained the EMG with bipolar surface electrodes, digitalized the electric potential with an $\mathrm{A} / \mathrm{D}$ converter, and analyzed the signals with bio-information multiuse analyzing program 'BIMUTAS ${ }^{\mathrm{TM}}$ ' (Kissei Medcomtec Co., Ltd., Nagano, Japan). 
In addition, a control study was performed on five normal people during walking or running using the same procedures as above.

\section{Calculation}

We estimated the IEMG by summing up the voltage using an A/D converter (sampling frequency: $500 \mathrm{~Hz}$ ). However, as the value became zero with simple summing, we used the absolute value of each voltage (ie, full wave rectification).

$$
\operatorname{IEMG}(\mathrm{mV} \cdot \mathrm{sec})=\sum|\mathrm{V}(\mathrm{t})(\mathrm{mV})|
$$

We also calculated the IGOM by the same method, and examined each value as follows.

(a) We compared the IGOM between the paraplegic patients and the control subjects over a period of $30 \mathrm{~s}(\mathrm{t}: 30 \mathrm{~s})$.

(b) We compared the average IEMG between the paraplegic patients during six cycles (at random) of cervical motion with goniometer and the control subjects during six-second motion (which was approximate to the time in case of paraplegic patients).

\section{Statistical analysis}

For the epidemiological investigation, the Student $t$ test was used for evaluation of differences between the two groups, and the chi-square test for examination of causal relationships. We predicted which variables (ie,

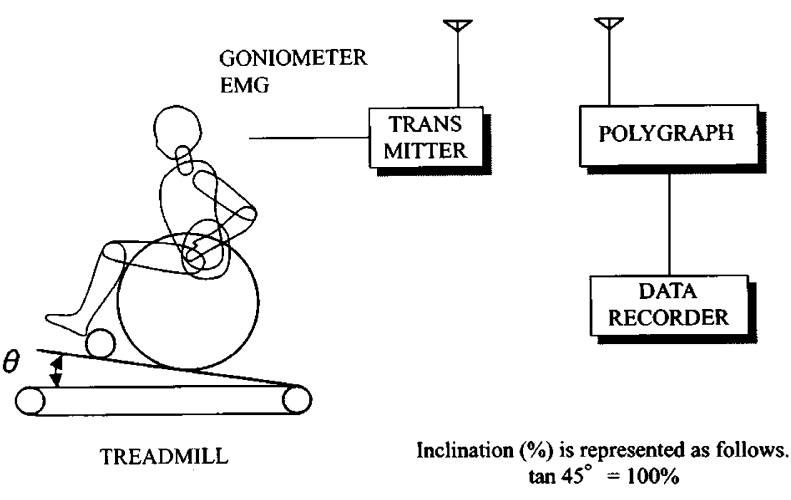

Figure 1 Schematic diagram for the system of movement analysis paraplegia, age, and duration) affected the incidence of CS by multivariate study using logistic regression analysis (SPSS Inc., Chicago, USA). For biomechanical examinations, we used the t-test for evaluation. Findings of $P<0.05$ were considered statistically significant.

\section{Results}

\section{Epidemiological investigation}

The results from the questionnaires were as follows. Fifty-six patients $(64 \%)$ had symptoms related to CS, and the incidence of symptomatic patients in the group with CS changes $(\mathrm{CS}+)(72 \%)$ was significantly higher than that in the group without $\mathrm{CS}$ changes $(\mathrm{CS}-)$ $(39 \%)$. CS + patients used the wheelchair for significantly longer periods of time everyday than did CS - patients, but the incidence of CS was not correlated with sports practice (Table 1).

The incidence of CS changes in the paraplegic paitents was higher than in the control group for all age groups, and these changes were related to age (Figure 2). CS changes were observed most frequently in the descending order of $\mathrm{C}_{5-6}(40 \%), \mathrm{C}_{6-7}(22 \%)$, and $\mathrm{C}_{4-5}(21 \%)$ (Figure 3). There was no difference in the radiographic features of CS changes between the two groups as previously reported. ${ }^{1,2}$ The topography of CS changes in the paraplegic patients is shown in Figure 4.

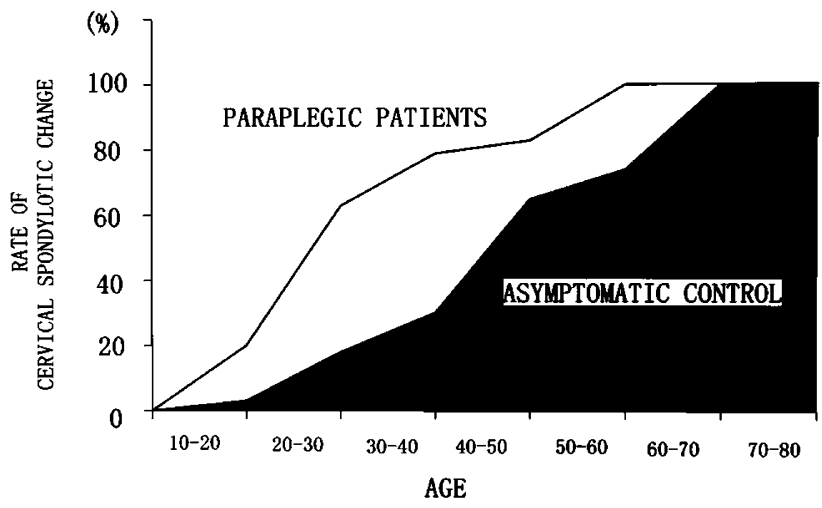

Figure 2 The incidence of cervical spondylosis in the paraplegic patients was higher than that in the control group for all age groups, and these changes were related to age

Table 1 Questionnaire results

\begin{tabular}{|c|c|c|c|c|c|c|c|c|c|}
\hline & $a$ & $\begin{array}{c}\text { Incic } \\
b\end{array}$ & $\begin{array}{cl}\mathrm{ce} & \mathrm{o} \\
\mathrm{c}\end{array}$ & $\begin{array}{l}\text { ach } \\
d\end{array}$ & $\begin{array}{c}n p t o \\
e\end{array}$ & $\left(\begin{array}{c}\% \\
f\end{array}\right)$ & $t$ & $\begin{array}{l}\text { Duration of use of } \\
\text { wheelchair (h/day) }\end{array}$ & $\begin{array}{l}\text { Engagements in } \\
\text { sports }(\%)\end{array}$ \\
\hline $\mathrm{CS}+(n=66)$ & 70 & 39 & 30 & 23 & 59 & 27 & $72^{-}$ & $10.4 \pm 4.4$ & $41-$ \\
\hline $\mathrm{CS}-(n=21)$ & 85 & 23 & 0 & 0 & 38 & 0 & 39 & $8.2 \pm 3.8$ & 48 \\
\hline
\end{tabular}

a: stiffness of neck or shoulder; b: neck pain; c: headache; d: back pain; e: numbness of upper extremity; f: motor weakness of the hand; t: total. $\mathrm{CS}+$ : group with $\mathrm{CS}$ changes; $\mathrm{CS}-$ : group without $\mathrm{CS}$ changes. ${ }^{*}$ chi-square test $P<0.01 ; * * t$-test $P<0.05$; $* * *$ chi-square test N.S. 
The incidence of CS changes (81\%) in patients with thoracolumbar injury (T11 L2) was higher than that $(74 \%)$ in patients with thoracic injury $(\mathrm{T} 1 \sim \mathrm{T} 10)$, but the difference was not significant. The mean ages in the two groups were approximately the same (thoracolumbar injury: $52.5 \pm 10.8$ years, thoracic injury: $50.2 \pm 11.1$ years), and the mean duration in the two groups were also approximately the same (thoracolumbar injury: $20.3 \pm 9.7$ years, thoracic injury: $16.4 \pm 10.9$ years).

Regression analysis showed that the probability of CS changes could be written as

$$
\text { Prob }(\text { CS changes })=1 /\left(1+e^{-z}\right)
$$

where

$$
Z=-5.5005+1.5630(\text { paraplegia })+0.1084(\text { age })
$$

Correlations were found between CS changes and paraplegia $(r=0.2064, P=0.0000)$, and between CS changes and age $(r=0.4034, P=0.0000)$. Using the above equation, the probability of $\mathrm{CS}$ changes in

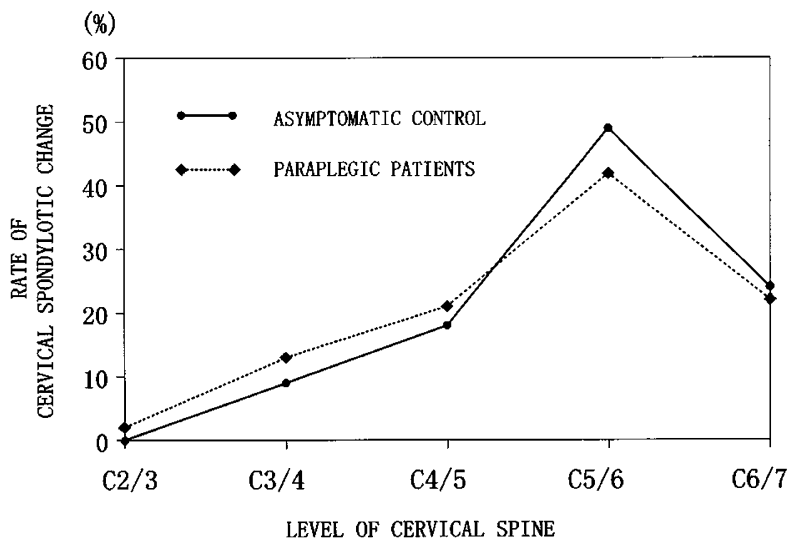

Figure 3 Cervical spondylosis occurred most frequently between the fifth and sixth cervical vertebrae in both the paraplegic patients and the normal control subjects

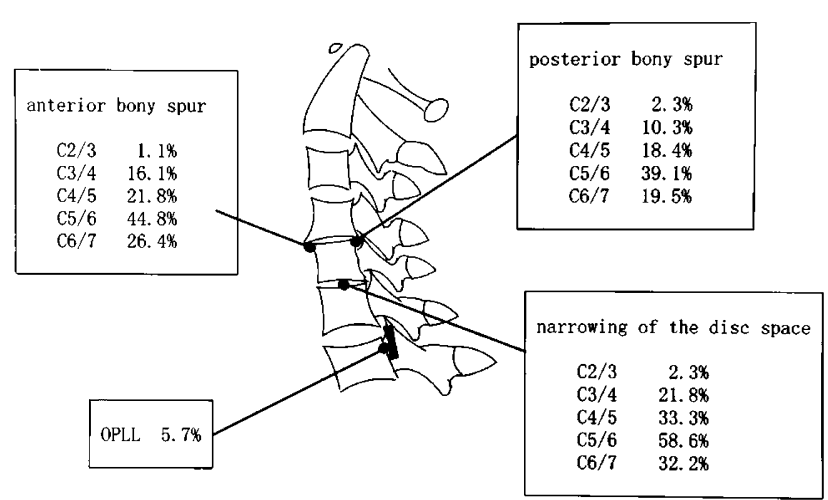

Figure 4 Topographic distribution of cervical spondylosis in the paraplegic patients $(n=87)$ paraplegic patients was found to be $2.36 \sim 9.66$ times that in normal controls, and the incidence was $1.09 \sim 1.14$ times increased with every year of increase in age. The odds of paraplegia were thus about 4.24 times against age.

In paraplegic patients, the linear combination $(\mathrm{Z})$ was

$$
Z=-3.0960+0.0370(\text { duration })+0.0778(\text { age })
$$

No correlation was found between CS changes and duration $(r=0.000, P=0.3089)$ but a correlation was found between CS changes and age $(r=0.2166$,

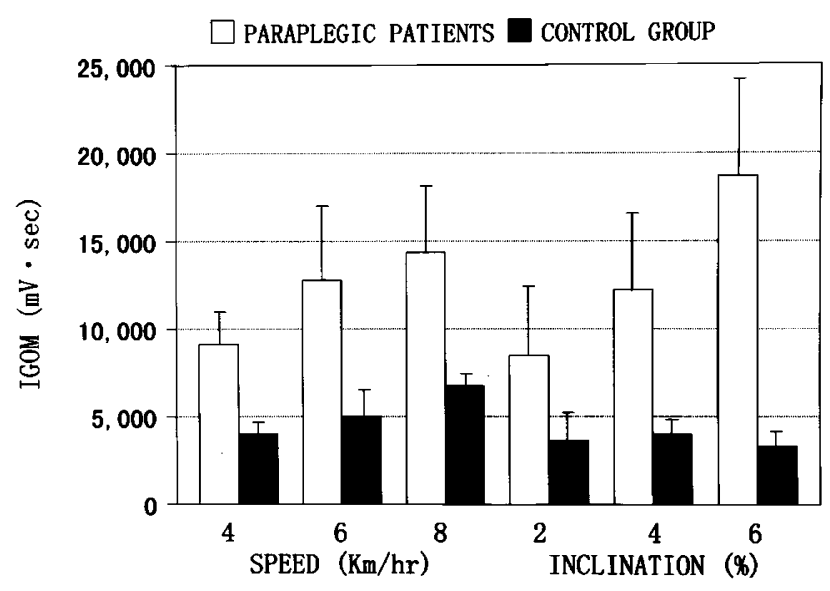

Figure 5 The IGOM was significantly larger in the paraplegic patients than in the control group at all stages $(P<0.01)$

\section{Sternocleidomastoid (right)}

Sternocleidomastoid
(left)

Trapezius

(right)

Trapezius

(left)

Goniometer
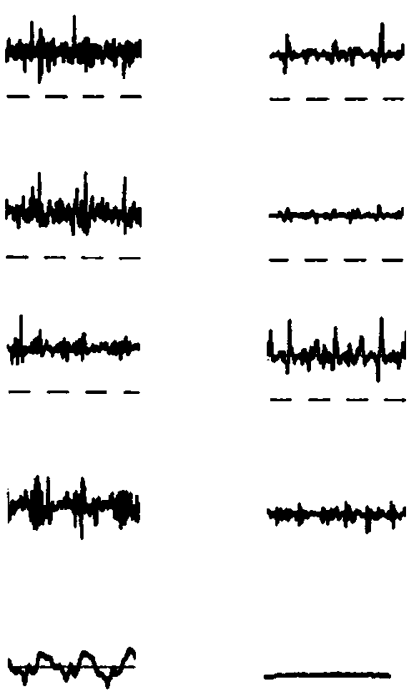

A
Figure 6 Subject A: 29-year-old paraplegic male patient (Th 11 ) at speed of $4 \mathrm{~km} / \mathrm{h}$ and inclination of $2 \%$. Subject B: $29-$ year-old normal male subject at speed of $4 \mathrm{~km} / \mathrm{h}$ and inclination of $2 \%$ 
$P=0.0131)$ ie, the incidence was $1.02 \sim 1.15$ times increased with every year of increase in age.

\section{Biomechanical examination}

(a) The IGOM in the paraplegic patients was significantly larger than that in the control group at all stages $(P<0.01)$, and tended to increase with increase in speed and inclination on testing (Figure 5). This indicated that the paraplegic patients frequently moved their cervical spine during wheelchair driving. Figure 6 shows the surface EMG and goniometry recorded in a paraplegic patient (A) and a normal control subject with normal gait (B). Subject A drove the wheelchair with frequent flexion and extension of his cervical spine, while subject B walked almost without moving his cervical spine.

(b) In the control group, the IEMG of the sternocleidomastoid muscle increased significantly with increase in speed $(P<0.001)$, and was significantly larger than in paraplegic patients at 6 and $8 \mathrm{Km} / \mathrm{h}(P<0.01)$. The changes of the sternocleidomastoid muscle IEMG with increase in inclination were significant in the paraplegic patients $(2-$ 4\%: $P<0.01,4-6 \%: P<0.05)$, but not in the control group (Figure 7).

The IEMG of the trapezius muscle also increased with increase in speed in the control group, and that of the control group was significantly larger than that of the paraplegic patients $(6 \mathrm{Km} / \mathrm{h}$ : $P<0.01,8 \mathrm{Km} / \mathrm{h}: P<0.001)$. However, in the paraplegic patients, the trapezius muscle IEMG significantly increased with increase in inclination $(2-4 \%: P<0.01,4-6 \%: P<0.05)$ and was larger than that of the control group, but not significantly (Figure 8).

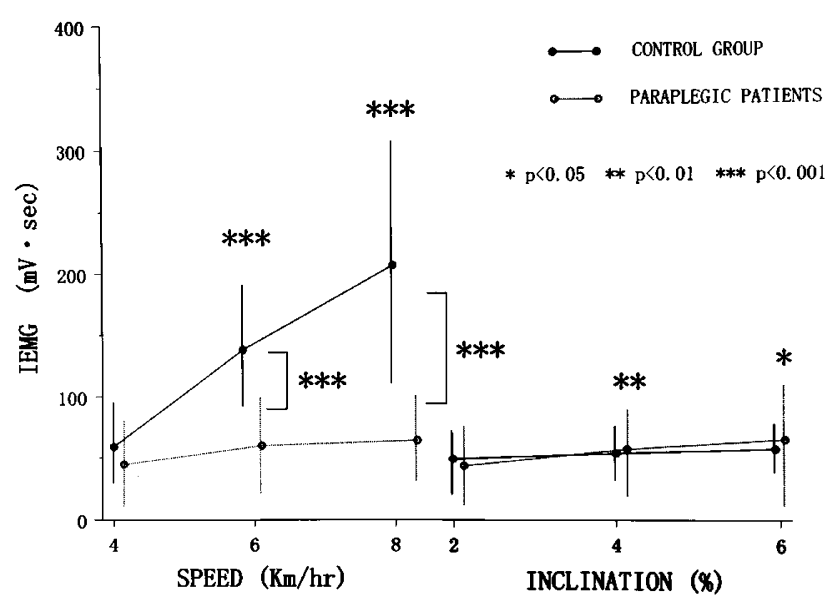

Figure 7 IEMG of the sternocleidomastoid muscle on increasing the speed and inclination. The IEMG of the control group significantly increased with increase in speed but not with increase in inclination. The IEMG of the paraplegic patients was vice versa

\section{Discussion}

Many authors have investigated the epidemiology of $\mathrm{CS},{ }^{1-5}$ but the CS changes have not so far been examined in paraplegic patients. Although the etiology of CS is unclear, many factors, such as genetic, injury, metabolic disorders, occupation, and posture are thought to be involved. The incidence of CS is said to show remarkable individual variations even in people of the same age and ethnic group.

We found that the incidence of $\mathrm{CS}$ in paraplegic patients was higher than that in normal subjects. Assuming the hypothesis that $\mathrm{CS}$ is caused by aging or biomechanical stress is true, we attributed the high incidence to the life style of paraplegic patients. Indeed our biomechanical analysis revealed that wheelchair driving imposed much load on the cervical spine.

It has previously been found that a linear relationship exists between IEMG and the tension during muscle contraction. ${ }^{6-8}$ However, it is difficult to use EMG for kinetic analysis, because when comparing various subjects, there are many differences in their skin conditions, muscle quality, and in the thickness of subcutaneous fat. Therefore, the IEMG is considered an index of the quantity of muscle activity itself under different conditions of mechanical efficiency, skin status, or muscle fatigue. ${ }^{7,9}$

Thorstensson et al. (1985) examined the EMG of the trunk muscles on performing voluntary movements, ${ }^{10}$ and Vitti et al. (1973) examined the EMG of the longus colli and sternocleidomastoid muscles during head-neck motions. ${ }^{11}$ Their studies demonstrated a basic pattern of alternation between the antagonist muscle groups and there was a sharp burst of activity on fast movement, while there were no activities in the resting positions.

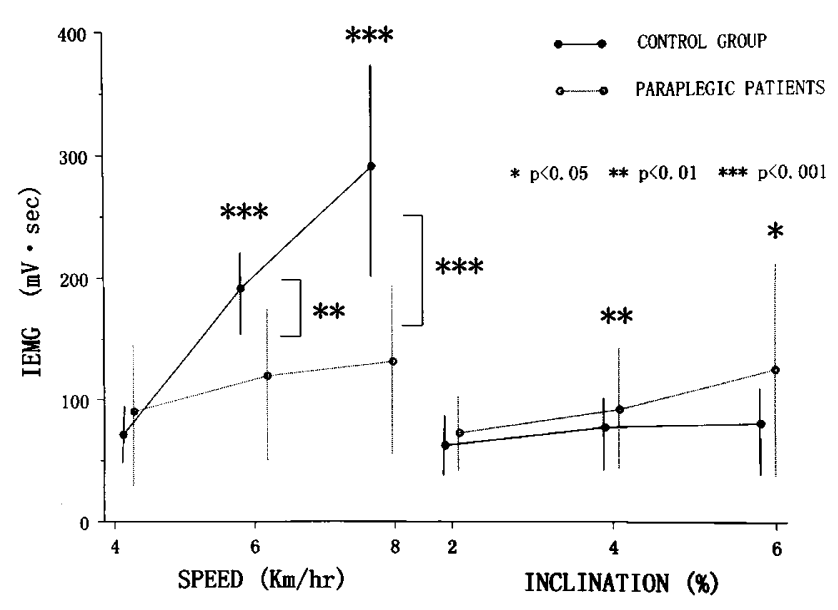

Figure 8 IEMG of the trapezius muscle on increasing the speed and inclination. The IEMG changes of the trapezius muscle were similar to those of the sternocleidomastoid muscle 


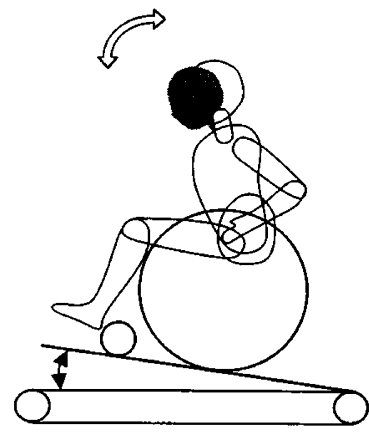

A

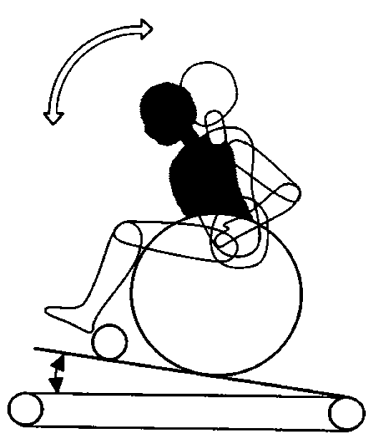

Figure 9 The schema of wheelchair driving action. (A) Injured spine was fixed with a long instrument. (B) Injured spine was not fixed or fixed by a short-fusion

In our series, the significantly high IGOM of paraplegic patients meant that they drove their wheelchair with frequent cervical motions. On the contrary, the IEMG in normal subjects increased with the increase of speed, and was greater than in paraplegic patients. We think that there was great muscle activity for holding the head during walking or running in spite of the limited cervical movements. We also found a large load on the cervical spine when increasing the speed in normal subjects and when increasing the inclination in paraplegic patients.

In the normal group, there were no significant individual differences when changing the speed and the inclination. However, in paraplegic patients, there were some individual IEMG changes on increasing the speed or the inclination. We thought that these changes are related to the subject's skill for wheelchair driving or to some conditions of the injured spine. If the patient fixed his injured spine with a long instrument, he drove the wheelchair with frequent cervical motions (Figure 9A). On the other hand, if he had a short fixation or no fixation, he drove the wheelchair with trunk motion but without cervical motion (Figure 9B).

The etiology of CS in paraplegic patients is probably not only the stress imposed by the frequent cervical motion during wheelchair driving, but we believe that this stress is one of the important causes of CS. Therefore, we should develop a new wheelchair which controls cervical motions, and teach patients a method of driving which imposes minimal or no cervical stress.

\section{References}

1 Friedenberg ZB, Miller WT. Degenerative disc disease of the cervical spine. J Bone Joint Surg 1963; 45A: $1171-1178$.

2 Kondou E, Andou K, Hattori S. Cervical osteochondrosis. J Jpn Orthop Assoc 1961; 35: 755 - 764 (in Japanese).

3 Horal J. The clinical appearance of low back disorders in the city of Gothenburg, Sweden. Acta Orthop Scandinavica 1969; Supplementum 118.

4 Kellgren JH, Lawrence JS, Bier F. Genetic factors in generalized osteoarthrosis. Ann Rheum Dis 1963; 22: 237 - 255.

5 DePalma A, Rothman R. The clinical syndrome of cervical disc disease. In: The Intervertebral Disc. W.B. Saunders: Philadelphia, 1970 , pp $85-91$.

6 Lippold OCJ. The relation between integrated action potentials in a human muscle and its isometric tension. J Physiol 1952; 117: $492-499$.

7 Lenman JAR. Quantitative electromyographic changes with muscular weakness. J Neurol Neurosurg Psychiat 1959; 22: $306-310$.

8 Henriksson J, Petersen FB. Integrated electromyography of quadriceps femoris muscle at different exercise intensities. $J \mathrm{Appl}$ Physiol 1974; 36: $218-220$.

9 Hiranaka T, Takeuchi K. Electromyographic findings in muscles around osteoarthritic knee: Integrated electromyography and frequency analysis. J Jpn Orthop Assoc 1995; 69: 675-684 (in Japanese).

10 Thorstensson A, Oddsson L, Carlson H. Motor control of voluntary trunk movements in standing. Acta Physiol Scandinavica 1985; 125: 309-321.

11 Basmajian JV, Carlo JDL. Muscles of mastication, face, and neck. In: Muscles Alive; Their functions revealed by electromyography. 5th ed. Williams and Wilkins: Baltimore 1985, pp $447-$ 469. 